\title{
From diffusion to reaction via $\Gamma$-convergence
}

\author{
Mark A. Peletier* \\ Giuseppe Savaré ${ }^{\dagger}$ \\ Marco Veneroni $i^{\ddagger}$
}

May 30, 2022

\begin{abstract}
We study the limit of high activation energy of a special Fokker-Planck equation, known as Kramers-Smoluchowski (K-S) equation. This equation governs the time evolution of the probability density of a particle performing a Brownian motion under the influence of a chemical potential $H / \varepsilon$. We choose $H$ having two wells corresponding to two chemical states $A$ and $B$. We prove that after a suitable rescaling the solution to (K-S) converges, in the limit of high activation energy $(\varepsilon \rightarrow 0)$, to the solution of a simple system modeling the diffusion of $A$ and $B$, and the reaction $A \rightleftharpoons B$.

The aim of this paper is to give a rigorous proof of Kramer's formal derivation and to embed chemical reactions and diffusion processes in a common variational framework which allows to derive the former as a singular limit of the latter, thus establishing a connection between two worlds often regarded as separate.

The singular limit is analysed by means of Gamma-convergence in the space of finite Borel measures endowed with the weak-* topology.

Key words and phrases: unification, scale-bridging, upscaling, high-energy limit, activation energy, Dirichlet forms, Mosco-convergence, variational evolution equations
\end{abstract}

AMS subject classification: 35K57, 35Q84, (49J45, 49S05, 80A30)

\section{Introduction}

\subsection{Chemical reaction as a diffusion process}

In a seminal paper in 1940, Hendrik Anthony Kramers described a number of approaches to the problem of calculating chemical reaction rates [12. One of the limit cases in this paper is equivalent to the motion of a Brownian particle in a (chemical) potential landscape. In this description a reaction event is the escape of the particle from one energy well into another.

This description is interesting for a number of reasons. It provides a connection between two processes, diffusion and reaction, which are often - especially at the macroscopic levelviewed as completely separate. It also provides a link between a macroscopic effect - chemical reaction - and a more microscopic, underlying motion, and in doing so, it highlights the fact that diffusion and reaction ultimately spring from the same underlying motion. It finally also allows for explicit calculation of reaction rates in terms of properties of the energy landscape.

In this paper we contribute to this discussion by studying the limit process of high activation energy in the unimolecular reaction $A \rightleftharpoons B$. As a first contribution, this provides a rigorous proof of the result that Kramers had derived formally. At the same time we extend his result to a Brownian motion in the product space spanned by both the chemical variable of Kramers and the variables corresponding to position in space, resulting in a limit system that models not only chemical reaction but also spatial diffusion-a simple reaction-diffusion system.

${ }^{*}$ Department of Mathematics and Institute for Complex Molecular Systems, Technische Universiteit Eindhoven, The Netherlands, m.a.peletier@tue.nl

${ }_{\dagger}$ Dipartimento di Matematica F.Casorati, Università degli studi di Pavia, Italy, giuseppe.savare@unipv.it

${ }^{\ddagger}$ Fakultät für Mathematik, Technische Universität Dortmund, Germany, marco.veneroni@math.uni-dortmund.de 
With this paper we have two aims. The first is to clarify the mathematical-rigorousaspects of the formal results of [12, and extend them to include spatial diffusion, and in this way to contribute to the upscaling of microscopic systems. The second is to make a first step in the construction of a variational framework that can describe the combination of general diffusive and chemically reactive processes. From this point of view it would be interesting, for example, to place the limit system in the context of Wasserstein gradient flows (see also Section 1.10. Initiated by the work of Otto [11, 15] and extended into many directions since, this framework provides an appealing variational structure for very general diffusion processes, but chemical reactions have so far resisted representation in the Wasserstein framework.

In this paper we only treat the simple equation $A \rightleftharpoons B$, but we plan to extend the approach to other systems in the future (see also [14]).

\subsection{The setup: enthalpy}

We consider the unimolecular reaction $A \rightleftharpoons B$. In chemical terms the $A$ and $B$ particles are two forms of the same molecule, such that the molecule can change from one form into the other. A typical example is a molecule with spatial asymmetry, which might exist in two distinct, mirror-image spatial configurations; another example is that of enzymes, for which the various spatial configurations also have different biological functions.

Remark. Classical, continuum-level modelling of the system of $A$ and $B$ particles that diffuse and react (see e.g. 9, 3]) leads to the set of differential equations, where we write $A$ and $B$ for the concentrations of $A$ and $B$ particles:

$$
\begin{aligned}
\partial_{t} A-D \Delta A & =k(B-A) \\
\partial_{t} B-D \Delta B & =k(A-B) .
\end{aligned}
$$

(See Section 1.10 for the equal reaction rates). This system will arise as the upscaling limit (see Theorem 1) of the system that we now develop in detail.

We next assume that the observed forms $A$ and $B$ correspond to the wells of an appropriate energy function. Since it is common in the chemical literature to denote by 'enthalpy difference' the release or uptake of heat as a particle $A$ is converted into a particle $B$, we shall adopt the same language and consider the $A$ and $B$ states to correspond to the wells of an enthalpy function $H$.

While the domain of definition of $H$ should be high-dimensional, corresponding to the many degrees of freedom of the atoms of the molecule, we will here make the standard reduction to a one-dimensional dependence. The variable $\xi$ is assumed to parametrize an imaginary 'optimal path' connecting the states $A$ to $B$, such that $\xi=-1$ corresponds to $A$ and $\xi=1$ to $B$. Such a path should pass through the 'mountain pass', the point which separates the basins of attraction of $A$ and $B$, and we arbitrarily choose that mountain pass to be at $\xi=0$, with $H(0)=1$. We also restrict $\xi$ to the interval $[-1,1]$, and we assume for simplicity that the wells are at equal depth, which we choose to be zero. A typical example of the function $H$ is showed in Figure 1

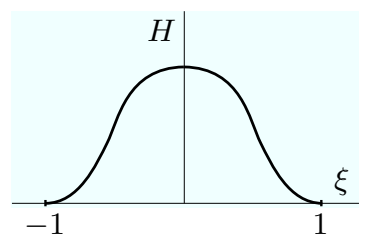

Figure 1: A typical function $H$

Specifically we make the following assumptions about $H: H \in C^{\infty}([-1,1]), H$ is even in $\xi$, maximal at $\xi=0$ with value 1 , and minimal at $\xi= \pm 1$ with value $0 ; H(\xi)>0$ for any $-1<\xi<1 ; H^{\prime}\left( \pm 1^{\mp}\right)=0$. The assumption of equal depth for the two wells corresponds to an assumption about the rate constants of the two reactions; we comment on this in Section 1.10 


\subsection{Diffusion in the chemical landscape}

This newly introduced 'chemical variable' $\xi$ should be interpreted as an internal degree of freedom of the particle, associated with internal changes in configuration. In the case of two alternative states of a molecule, $\xi$ parametrizes all the intermediate states along a connecting path.

In this view the total state of a particle consists of this chemical state $\xi$ together with the spatial position of the particle, represented by a $d$-dimensional spatial variable $x$ in a Lipschitz, bounded, and open domain $\Omega \subset \mathbb{R}^{d}$, so that the full state space for the particle is the closure $\bar{D}$ of

$$
D:=\Omega \times(-1,1) \quad \text { with variables }(x, \xi) .
$$

Taking a probabilistic point of view, and following Kramers, the motion of the particle will be described in terms of its probability density $\rho \in \mathscr{P}(\bar{D})$, in the sense that for Borel sets $X \subset \bar{\Omega}$ and $\Xi \subset[-1,1]$ the number $\rho(X \times \Xi)$ is the probability of finding the particle at a position $x \in X$ and with a 'chemical state' $\xi \in \Xi$.

The particle is assumed to perform a Brownian motion in $D$, under the influence of the potential landscape described by $H$. This assumption corresponds to the 'large-friction limit' discussed by Kramers. The time evolution of the probability distribution $\rho$ then is given by the Kramers-Smoluchowski equation

$$
\partial_{t} \rho-\Delta_{x} \rho-\tau \partial_{\xi}\left(\partial_{\xi} \rho+\rho \partial_{\xi} H\right)=0 \quad \text { in } \mathscr{D}^{\prime}(D \times(0, \infty)),
$$

with Neumann boundary conditions on the lateral boundary $\partial D$. The coefficient $\tau>0$ is introduced to parametrize the difference in scales for $x$ and $\xi$ : since $x$ is a rescaled physical distance, and $\xi$ is a rescaled 'chemical' distance, the units of length in the two variables are different, and the parameter $\tau$ can be interpreted as the factor that converts between the two scales. Below we shall make an explicit choice for $\tau$.

\subsection{The limit of high activation energy}

In the setup as described above, there is a continuum of states (i.e. $(-1,1)$ ) connecting the $A$ state to the $B$ state, and a statement of the type 'the particle is in the $A$ state' is therefore not well defined. In order to make a connection with the macroscopic description ' $A \rightleftharpoons B$ ', which presupposes a clear distinction between the two states, we take the limit of high activation energy, as follows.

We rescale the enthalpy $H$ with a small parameter $\varepsilon$, to make it $H(\xi) / \varepsilon$. (This is called 'high activation energy' since $\max _{\xi} H(\xi) / \varepsilon=1 / \varepsilon$ is the height of the mountain that a particle has to climb in order to change states).

This rescaling has various effects on the behaviour of solutions $\rho$ of (2). To illustrate one effect, let us consider the invariant measure $\gamma_{\varepsilon}$, the unique stationary solution in $\mathscr{P}(\bar{D})$ of $[2]$ :

$$
\gamma_{\varepsilon}=\lambda_{\Omega} \otimes \tilde{\gamma}_{\varepsilon}, \quad \lambda_{\Omega}:=\left.\frac{1}{\mathcal{L}^{d}(\Omega)} \mathcal{L}^{d}\right|_{\Omega}, \quad \tilde{\gamma}_{\varepsilon}=\left.Z_{\varepsilon}^{-1} \mathrm{e}^{-H / \varepsilon} \mathcal{L}^{1}\right|_{[-1,1]}
$$

(where $\mathcal{L}^{1}, \mathcal{L}^{d}$ are the 1 - and $d$-dimensional Lebesgue measures). The constant $Z_{\varepsilon}$ is fixed by the requirement that $\gamma_{\varepsilon}(\bar{D})=\tilde{\gamma}_{\varepsilon}([-1,1])=1$.

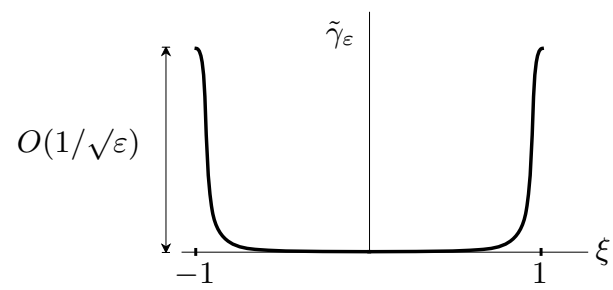

Figure 2: The density $\tilde{\gamma}_{\varepsilon}$

Since $H$ is strictly positive at any $-1<\xi<1$, the exponential $\exp (-H(\xi) / \varepsilon)$ vanishes at all $\xi$ except for $\xi= \pm 1$; therefore the measure $\gamma_{\varepsilon}$ concentrates on the lines $\xi=-1$ and $\xi=1$, 
and converges weakly-* as $\varepsilon \rightarrow 0$ to the limit measure $\gamma$ given by

$$
\gamma=\lambda_{\Omega} \otimes \tilde{\gamma}, \quad \tilde{\gamma}:=\frac{1}{2}\left(\delta_{-1}+\delta_{1}\right)
$$

Here weak-* convergence is to be interpreted in the duality with continuous functions in $\bar{D}$ (thus considering $\mathscr{P}(\bar{D})$ as a weakly-* closed convex subset of the space $\mathscr{M}(\bar{D})=\left(\mathrm{C}^{0}(\bar{D})\right)^{\prime}$ of signed Borel measures with finite total variation) i.e.

$\lim _{\varepsilon \rightarrow 0} \int_{\bar{D}} \phi(x, \xi) \mathrm{d} \gamma_{\varepsilon}=\int_{\bar{D}} \phi(x, \xi) \mathrm{d} \gamma(x, \xi)=\frac{1}{2} \int_{\Omega}(\phi(x,-1)+\phi(x, 1)) \mathrm{d} \lambda_{\Omega}(x), \quad$ for any $\phi \in \mathrm{C}^{0}(\bar{D})$.

We should interpret the behaviour of $\gamma_{\varepsilon}$ as follows. In the limit $\varepsilon \rightarrow 0$, the deep wells at $\xi= \pm 1$ force particles to stay increasingly close to the bottom of the wells. However, at any given $\varepsilon>0$, there is a positive probability that a particle switches from one well to the other in any given period of time. The rate at which this happens is governed by the local structure of $H$ near $\xi= \pm 1$ and near $\xi=0$, and becomes very small-of order $\varepsilon^{-1} \exp (-1 / \varepsilon)$, as we shall see below.

In the limit $\varepsilon=0$, the behaviour of particles in the $\xi$-direction is no longer recognizable as diffusional in nature. In the $\xi$-direction a particle can only be in one of two states $\xi= \pm 1$, which we therefore interpret as the $A$ and $B$ states. Of the diffusional movement in the $\xi$ direction only a jump process remains, in which a particle at $\xi=-1$ jumps with a certain rate to position $\xi=1$, or vice versa.

\subsection{Spatiochemical rescaling}

Since the jumping (chemical reaction) rate at finite $\varepsilon>0$ is of order $\varepsilon^{-1} \exp (-1 / \varepsilon)$, the limiting reaction rate will be zero unless we rescale the system appropriately. This requires us to speed up time by a factor of $\varepsilon \exp (1 / \varepsilon)$. At the same time, the diffusion rate in the $x$-direction remains of order 1 as $\varepsilon \rightarrow 0$, and the rescaling should preserve this. In order to obtain a limit in which both diffusion in $x$ and chemical reaction in $\xi$ enter at rates that are of order 1 , we use the freedom of choosing the parameter $\tau$ that we introduced above.

We therefore choose $\tau$ equal to

$$
\tau_{\varepsilon}:=\varepsilon \exp (1 / \varepsilon)
$$

and we then find the differential equation

$$
\partial_{t} \rho_{\varepsilon}-\Delta_{x} \rho_{\varepsilon}-\tau_{\varepsilon} \partial_{\xi}\left(\partial_{\xi} \rho_{\varepsilon}+\frac{1}{\varepsilon} \rho_{\varepsilon} \partial_{\xi} H\right)=0 \quad \text { in } \mathscr{D}^{\prime}(D \times(0, \infty)),
$$

which clearly highlights the different treatment of $x$ and $\xi$ : the diffusion in $x$ is independent of $\tau_{\varepsilon}$ while the diffusion and convection in the $\xi$-variable are accelerated by a factor $\tau_{\varepsilon}$.

\subsection{Switching to the density variable}

As is already suggested by the behaviour of the invariant measure $\gamma_{\varepsilon}$, the solution $\rho_{\varepsilon}$ will become strongly concentrated at the extremities $\{ \pm 1\}$ of the $\xi$-domain $(-1,1)$. This is the reason why it is useful to interpret $\rho_{\varepsilon}$ as a family $\rho_{\varepsilon}(t, \cdot)$ of time-dependent measures, instead of functions. It turns out that the densities $u_{\varepsilon}(t, \cdot)$

$$
u_{\varepsilon}(t, \cdot):=\frac{\mathrm{d} \rho(t, \cdot)}{\mathrm{d} \gamma_{\varepsilon}}
$$

of $\rho_{\varepsilon}(t, \cdot)$ with respect to $\gamma_{\varepsilon}$ also play a crucial role and it is often convenient to have both representations at our disposal, freely switching between them. In terms of the variable $u_{\varepsilon}$ equation (6) becomes

$$
\partial_{t} u_{\varepsilon}-\Delta_{x} u_{\varepsilon}-\tau_{\varepsilon}\left(\partial_{\xi \xi}^{2} u_{\varepsilon}-\frac{1}{\varepsilon} \partial_{\xi} H \partial_{\xi} u_{\varepsilon}\right)=0 \quad \text { in }(0,+\infty) \times D,
$$

supplemented with the boundary conditions

$$
\partial_{\xi} u_{\varepsilon}(t, x, \pm 1)=0 \quad \text { for all } x \in \Omega, \quad \nabla_{x} u_{\varepsilon}(t, x, \xi) \cdot \mathbf{n}=0 \quad \text { on } \partial \Omega \times[-1,1], \quad t>0 .
$$


We choose an initial condition

$$
u_{\varepsilon}(0, x, \xi)=u_{\varepsilon}^{0}(x, \xi), \quad \text { for all }(x, \xi) \in D, \quad \text { with } \quad \rho_{\varepsilon}^{0}=u_{\varepsilon}^{0} \gamma_{\varepsilon} \in \mathscr{P}(\bar{D}) .
$$

Let us briefly say something about the functional-analytic setting. It is well known (see e.g. [7]) that the operator $A_{\varepsilon}:=-\Delta_{x}-\tau_{\varepsilon} \partial_{\xi \xi}^{2}+\left(\tau_{\varepsilon} / \varepsilon\right) H^{\prime} \partial_{\xi}$ with Neumann boundary conditions (8) has a self-adjoint realization in the space $H_{\varepsilon}:=L^{2}\left(D ; \gamma_{\varepsilon}\right)$. Therefore the weak form of equation (7) can be written as

$$
b_{\varepsilon}\left(\partial_{t} u(t), v\right)+a_{\varepsilon}(u(t), v)=0 \quad \text { for all } v \in V_{\varepsilon},
$$

where the bilinear forms $a_{\varepsilon}$ and $b_{\varepsilon}$ are defined by

$$
b_{\varepsilon}: H_{\varepsilon} \times H_{\varepsilon} \rightarrow \mathbb{R}, \quad b_{\varepsilon}(u, v):=\int_{D} u v \mathrm{~d} \gamma_{\varepsilon}
$$

and

$$
\begin{aligned}
& V_{\varepsilon}:=W^{1,2}\left(D ; \gamma_{\varepsilon}\right):=\left\{u \in L^{2}\left(D, \gamma_{\varepsilon}\right) \cap W_{\mathrm{loc}}^{1,1}(D): \int_{D}\left|\nabla_{x, \xi} u\right|^{2} \mathrm{~d} \gamma_{\varepsilon}<+\infty\right\}, \\
& a_{\varepsilon}: V_{\varepsilon} \times V_{\varepsilon} \rightarrow \mathbb{R}, \quad a_{\varepsilon}(u, v):=\int_{D} A_{\varepsilon} u v \mathrm{~d} \gamma_{\varepsilon}=\int_{D}\left(\nabla_{x} u \nabla_{x} v+\tau_{\varepsilon} \partial_{\xi} u_{\varepsilon} \partial_{\xi} v\right) \mathrm{d} \gamma_{\varepsilon} .
\end{aligned}
$$

Since $V_{\varepsilon}$ is densely and continuously imbedded in $H_{\varepsilon}$, standard results on variational evolution equations in an Hilbert triplet (see e.g. 13, 6]) and their regularizing effects show that a unique solution exists in $C\left([0, \infty) ; H_{\varepsilon}\right) \cap C^{\infty}\left((0, \infty) ; V_{\varepsilon}\right)$ for every initial datum $u_{\varepsilon}^{0} \in H_{\varepsilon}$.

\subsection{Main result I: weak convergence of $\rho_{\varepsilon}$ and $u_{\varepsilon}$}

The following theorem is the first main result of this paper. It states that for every time $t \geq 0$ the measures $\rho_{\varepsilon}(t)$ solutions of $(6)$ weakly-* converge to a limiting measure $\rho(t)$ in $\mathscr{P}(\bar{D})$, whose density $u(t)=\frac{\mathrm{d} \rho(t)}{\mathrm{d} \gamma}$ is the solution of the limit system (1). Note that for a function $u \in L^{2}(\bar{D}, \gamma)$ the traces $u^{ \pm}=u(\cdot, \pm 1) \in L^{2}(\Omega)$ are well defined (in fact, the map $u \mapsto\left(u^{-}, u^{+}\right)$is an isomorphism between $L^{2}(\bar{D}, \gamma)$ and $\left.L^{2}\left(\Omega, \frac{1}{2} \lambda_{\Omega} ; \mathbb{R}^{2}\right)\right)$.

We state our result in a general form, which holds even for signed measures in $\mathscr{M}(\bar{D})$.

Theorem 1. Let $\rho_{\varepsilon}=u_{\varepsilon} \gamma_{\varepsilon} \in \mathrm{C}^{0}([0,+\infty) ; \mathscr{M}(\bar{D}))$ be the solution of $(6$ 5) with initial datum $\rho_{\varepsilon}^{0}$. If

$$
\sup _{\varepsilon>0} \int_{D}\left|u_{\varepsilon}^{0}\right|^{2} \mathrm{~d} \gamma_{\varepsilon}<+\infty
$$

and

$$
\rho_{\varepsilon}^{0} \text { weakly-* converges to } \rho^{0}=u^{0} \gamma=\frac{1}{2} u^{0-} \lambda_{\Omega} \otimes \delta_{-1}+\frac{1}{2} u^{0+} \lambda_{\Omega} \otimes \delta_{+1} \text { as } \varepsilon \downarrow 0,
$$

then $u^{0} \in L^{2}(\bar{D}, \gamma), u^{0, \pm} \in L^{2}(\Omega)$, and for every $t \geq 0$ the solution $\rho_{\varepsilon}(t)$ weakly-* converge to

$$
\rho(t)=u(t) \gamma=\frac{1}{2} u^{-}(t) \lambda_{\Omega} \otimes \delta_{-1}+\frac{1}{2} u^{+}(t) \lambda_{\Omega} \otimes \delta_{+1},
$$

whose densities $u^{ \pm}$belong to $C^{0}\left([0,+\infty) ; L^{2}(\Omega)\right) \cap C^{1}\left((0,+\infty) ; W^{1,2}(\Omega)\right)$ and solve the system

$$
\begin{array}{ll}
\partial_{t} u^{+}-\Delta_{x} u^{+}=k\left(u^{-}-u^{+}\right) & \text {in } \Omega \times(0,+\infty) \\
\partial_{t} u^{-}-\Delta_{x} u^{-}=k\left(u^{+}-u^{-}\right) & \text {in } \Omega \times(0,+\infty) \\
u^{ \pm}(0)=u^{0, \pm} & \text { in } \Omega .
\end{array}
$$

The positive constant $k$ in $(14 \mathrm{a}, \mathrm{b})$ can be characterized as the asymptotic minimal transition cost

$$
k=\frac{1}{\pi} \sqrt{\left|H^{\prime \prime}(0)\right| H^{\prime \prime}(1)}=\lim _{\varepsilon \downarrow 0} \min \left\{\tau_{\varepsilon} \int_{-1}^{1}\left(\varphi^{\prime}(\xi)\right)^{2} \mathrm{~d} \tilde{\gamma}_{\varepsilon}: \varphi \in W^{1,2}(-1,1), \varphi( \pm 1)= \pm \frac{1}{2}\right\} .
$$


Remark (The variational structure of the limit problem). The " $\varepsilon=0$ " limit problem 14a $14 \mathrm{c}$ admits the same variational formulation of the " $\varepsilon>0$ " problem we introduced in Section 1.6. Recall that $\gamma$ is the measure defined in (4) as the weak limit of $\gamma_{\varepsilon}$; we set $H:=L^{2}(D, \gamma)$, and for every $\rho=u \gamma$ with $u \in H$ we set $u^{ \pm}(x):=u(x, \pm 1) \in L^{2}\left(\Omega, \lambda_{\Omega}\right)$. We define

$$
b(u, v):=\int_{D} u(x, \xi) v(x, \xi) \mathrm{d} \gamma(x, \xi)=\frac{1}{2} \int_{\Omega}\left(u^{+} v^{+}+u^{-} v^{-}\right) \mathrm{d} \lambda_{\Omega} .
$$

Similarly, we set $V:=\left\{u \in H: u^{ \pm} \in W^{1,2}(\Omega)\right\}$, which is continuously and densely imbedded in $H$, and

$$
a(u, v):=\frac{1}{2} \int_{\Omega}\left(\nabla_{x} u^{+} \nabla_{x} v^{+}+\nabla_{x} u^{-} \nabla_{x} v^{-}+k\left(u^{+}-u^{-}\right)\left(v^{+}-v^{-}\right)\right) \mathrm{d} \lambda_{\Omega} .
$$

Then the system $(14 a, b, c)$ can be formulated as

$$
b\left(\partial_{t} u(t), v\right)+a(u(t), v)=0 \quad \text { for every } t>0 \text { and } v \in V,
$$

which has the same structure as 10 .

\subsection{Main result II: a stronger convergence of $u_{\varepsilon}$}

Weak-* convergence in the sense of measures is a natural choice in order to describe the limit of $\rho_{\varepsilon}$, since the densities $u_{\varepsilon}$ and the limit density $u=\left(u^{+}, u^{-}\right)$are defined on different domains with respect to different reference measures. Nonetheless it is possible to consider a stronger convergence which better characterizes the limit, and to prove that it is satisfied by the solutions of our problem.

This stronger notion is modeled on Hilbert spaces (or, more generally, on Banach spaces with a locally uniformly convex norm), where strong convergence is equivalent to weak convergence together with the convergence of the norms:

$$
x_{n} \rightarrow x \quad \Longleftrightarrow \quad x_{n} \rightarrow x \quad \text { and } \quad\left\|x_{n}\right\| \rightarrow\|x\| .
$$

In this spirit, the next result states that under the additional request of "strong" convergence of the initial data $u_{\varepsilon}^{0}$, we have "strong" convergence of the densities $u_{\varepsilon}$; we refer to [17, 10. (see also [2, Sec. 5.4]) for further references in a measure-theoretic setting.

Theorem 2. Let $\rho_{\varepsilon}, \rho_{\varepsilon}^{0}$ be as in Theorem 1. If moreover

$$
\lim _{\varepsilon \downarrow 0} b_{\varepsilon}\left(u_{\varepsilon}^{0}, u_{\varepsilon}^{0}\right)=b\left(u^{0}, u^{0}\right)
$$

then for every $t>0$ we have

$$
\lim _{\varepsilon \downarrow 0} b_{\varepsilon}\left(u_{\varepsilon}(t), u_{\varepsilon}(t)\right)=b(u(t), u(t))
$$

and

$$
\lim _{\varepsilon \downarrow 0} a_{\varepsilon}\left(u_{\varepsilon}(t), u_{\varepsilon}(t)\right)=a(u(t), u(t)) .
$$

Applying, e.g., 2], Theorem 5.4.4] we can immediately deduce the following result, which clarifies the strengthened form of convergence that we are considering here. This convergence is strong enough to allow us to pass to the limit in nonlinear functions of $u_{\varepsilon}$ :

Corollary 3. Under the same assumptions as in Theorem 2 we have

$$
\begin{aligned}
\lim _{\varepsilon \downarrow 0} \int_{D} f(x, \xi, & \left.u_{\varepsilon}(x, \xi, t)\right) \mathrm{d} \gamma_{\varepsilon}(x, \xi)=\int_{\bar{D}} f(x, \xi, u(x, \xi, t)) \mathrm{d} \gamma(x, \xi) \\
& =\frac{1}{2} \int_{\Omega}\left(f\left(x,-1, u^{-}(x, t)\right)+f\left(x, 1, u^{+}(x, t)\right)\right) \mathrm{d} \lambda_{\Omega}(x) \quad \text { for every } t>0,
\end{aligned}
$$

where $f: \bar{D} \times \mathbb{R} \rightarrow \mathbb{R}$ is an arbitrary continuous function satisfying the quadratic growth condition

$$
|f(x, \xi, r)| \leq A+B r^{2} \quad \text { for every }(x, \xi) \in \bar{D}, r \in \mathbb{R}
$$

for suitable nonnegative constants $A, B \in \mathbb{R}$. 


\subsection{Structure of the proof}

Let us briefly explain the structure of the proof of Theorems 1 and 2 This will also clarify the term $\Gamma$-convergence in the title, and highlight the potential of the method for wider application.

The analogy between $(10)$ and $(18)$ suggests to pass to the limit in these weak formulations, or even better, in their equivalent integrated forms

$$
b_{\varepsilon}\left(u_{\varepsilon}(t), v_{\varepsilon}\right)+\int_{0}^{t} a_{\varepsilon}\left(u_{\varepsilon}(t), v_{\varepsilon}\right) \mathrm{d} t=b\left(u_{\varepsilon}^{0}, v_{\varepsilon}\right), \quad b(u(t), v)+\int_{0}^{t} a(u(t), v) \mathrm{d} t=b\left(u^{0}, v\right) .
$$

Applying standard regularization estimates for the solutions to 10 and a weak coercivity property of $b_{\varepsilon}$, it is not difficult to prove that $u_{\varepsilon}(t)$ "weakly" converges to $u(t)$ for every $t>0$, i.e.

$$
\rho_{\varepsilon}(t)=u_{\varepsilon}(t) \gamma_{\varepsilon} \rightarrow \rho(t)=u(t) \gamma \quad \text { weakly-* in } \mathscr{M}(\bar{D}) .
$$

The concept of weak convergence of densities that we are using here is thus the same as in Theorem 1, i.e. weak-* convergence of the corresponding measures in $\mathscr{M}(\bar{D})$.

In order to pass to the limit in 24 the central property is the following weak-strong convergence principle:

For every $v \in V$ there exists $v_{\varepsilon} \in V_{\varepsilon}$ with $v_{\varepsilon} \rightarrow v$ as $\varepsilon \rightarrow 0$ such that for every $u_{\varepsilon} \rightarrow u$

$$
b_{\varepsilon}\left(u_{\varepsilon}, v_{\varepsilon}\right) \rightarrow b(u, v) \quad \text { and } \quad a_{\varepsilon}\left(u_{\varepsilon}, v_{\varepsilon}\right) \rightarrow a(u, v) .
$$

Note that the previous property implies in particular that recovery family $v_{\varepsilon}$ converges "strongly" to $v$, according to the notion considered by Theorem 2 i.e. $v_{\varepsilon} \rightarrow v$ iff $v_{\varepsilon} \rightarrow v$ with both $b_{\varepsilon}\left(v_{\varepsilon}, v_{\varepsilon}\right) \rightarrow b(v, v)$ and $a_{\varepsilon}\left(v_{\varepsilon}, v_{\varepsilon}\right) \rightarrow a(v, v)$. Corollary 6 shows that this weak-strong convergence property can be derived from $\Gamma$-convergence in the "weak" topology of the family of quadratic forms

$$
q_{\varepsilon}^{\kappa}(u):=b_{\varepsilon}(u, u)+\kappa a_{\varepsilon}(u, u) \quad \text { to } \quad q^{\kappa}(u):=b(u, u)+\kappa a(u, u) \text { for } \kappa>0 .
$$

In order to formulate this property in the standard framework of $\Gamma$-convergence we will extend $a_{\varepsilon}$ and $b_{\varepsilon}$ to lower semi-continuous quadratic functionals (possibly assuming the value $+\infty$ ) in the space $\mathscr{M}(\bar{D})$, following the approach of [8, Chap. 11-13]. While the $\Gamma$-convergence of $b_{\varepsilon}$ is a direct consequence of the weak convergence of $\gamma_{\varepsilon}$ to $\gamma$, the convergence of $a_{\varepsilon}$ is more subtle. The convergence of $a_{\varepsilon}$ and the structure of the limit depends critically on the choice of $\tau_{\varepsilon}$ (defined in (5)): as we show in Section 3.2, the scaling of $\tau_{\varepsilon}$ in terms of $\varepsilon$ is chosen exactly such that the strength of the 'connection' between $\xi=-1$ and $\xi=1$ is of order $O(1)$ as $\varepsilon \rightarrow 0$.

The link between $\Gamma$-convergence and stability of evolution problems of parabolic type is well known when $b_{\varepsilon}=b$ is a fixed and coercive bilinear form (see, e.g., [4, Chap. 3.9.2]) and can therefore be considered as the scalar product of the Hilbert space $H_{\varepsilon} \equiv H$. In this case the embedding of the problems in a bigger topological vector space (the role played by $\mathscr{M}(\bar{D})$ in our situation) is no more needed, and one can deal with the weak and strong topology of $H$, obtaining the following equivalent characterizations (see e.g. 5, Th. 3.16] and [8, Th. 13.6]):

1. Pointwise (strong) convergence in $H$ of the solutions of the evolution problems;

2. Pointwise convergence in $H$ of the resolvents of the linear operators associated to the bilinear forms $a_{\varepsilon}$;

3. Mosco-convergence in $H$ of the quadratic forms associated to $a_{\varepsilon}$;

4. $\Gamma$-convergence in the weak topology of $H$ of the quadratic forms $b+\kappa a_{\varepsilon}$ to $b+\kappa a$ for every $\kappa>0$.

In the present case, where $b_{\varepsilon}$ does depend on $\varepsilon, \Gamma$-convergence of the extended quadratic forms $b_{\varepsilon}+\kappa a_{\varepsilon}$ with respect to the weak-* topology of $\mathscr{M}(\bar{D})$ is thus a natural extension of the latter condition; Theorem 4 can be interpreted as essentially proving a slightly stronger version of this property. Starting from this $\Gamma$-convergence result, we will derive the convergence of the evolution problems by a simple and general argument, which we will present in Section 4 


\subsection{Discussion}

The result of Theorem 1 is amongst other things a rigorous version of the result of Kramers 12 that was mentioned in the introduction. It shows that the simple reaction-diffusion system (14) can indeed be viewed as an upscaled version of a diffusion problem in an augmented phase space; or, equivalently, as an upscaled version of the movement of a Brownian particle in the same augmented phase space.

At the same time it generalizes the work of Kramers by adding the spatial dimension, resulting in a limit system which - for this choice of $\tau_{\varepsilon}$, see below for more on this choicecaptures both reaction and diffusion effects.

Measures versus densities. It is interesting to note the roles of the measures $\rho_{\varepsilon}, \rho$ and their densities $u_{\varepsilon}, u$ with respect to $\gamma_{\varepsilon}, \gamma$. The variational formulation of the equations are done in terms of the densities $u_{\varepsilon}, u$ but the limit procedure is better understood in terms of the measures $\rho_{\varepsilon}, \rho$, since a weak-* convergence is involved. This also allows for a unification of two problems with a different structure (a Fokker-Planck equation for $u_{\varepsilon}$ and a reaction-diffusion system for the couple $u^{-}, u^{+}$.)

Gradient flows. The weak formulation 10 shows also that a solution $u_{\varepsilon}$ can be interpreted as a gradient flow of the quadratic energy $\frac{1}{2} a_{\varepsilon}(u, u)$ with respect to the $L^{2}\left(D, \gamma_{\varepsilon}\right)$ distance. Another gradient flow structure for the solutions of the same problem could be obtained by a different choice of energy functional and distance: for example, as proved in 11, FokkerPlanck equations like (6) can be interpreted also as the gradient flow of the relative entropy functional

$$
\mathcal{H}\left(\rho \mid \gamma_{\varepsilon}\right):=\int_{D} \frac{\mathrm{d} \rho}{\mathrm{d} \gamma_{\varepsilon}} \log \left(\frac{\mathrm{d} \rho}{\mathrm{d} \gamma_{\varepsilon}}\right) \mathrm{d} \gamma_{\varepsilon}
$$

in the space $\mathscr{P}(\bar{D})$ of probability measures endowed with the so-called $L^{2}$-Wasserstein distance (see e.g. [2]). Other recent work [1] suggests that the Wasserstein setting can be the most natural for understanding diffusion as a limit of the motion of Brownian particles, but in this case it is not obvious how to interpret the limit system in the framework of gradient flows on probability measures, and how to obtain it in the limit as $\varepsilon \rightarrow 0$.

In a forthcoming paper we investigate a new distance for the limit problem, modeled on the reaction-diffusion term, and we study how the limit couple of energy and dissipation can be obtained as a $\Gamma$-limit.

The choice of $\tau_{\varepsilon}$. In this paper the time scale $\tau_{\varepsilon}$ is chosen to be equal to $\varepsilon \exp (1 / \varepsilon)$, and it is a natural question to ask about the limit behaviour for different choices of $\tau_{\varepsilon}$. If the scaling is chosen differently-i.e. if $\tau_{\varepsilon} \varepsilon^{-1} \exp (-1 / \varepsilon)$ converges to 0 or $\infty$ - then completely different limit systems are obtained:

- If $\tau_{\varepsilon} \ll \varepsilon \exp (1 / \varepsilon)$, then the reaction is not accelerated sufficiently as $\varepsilon \rightarrow 0$, and the limit system will contain only diffusion (i.e. $k=0$ in $(14)$ );

- If $\tau_{\varepsilon} \gg \varepsilon \exp (1 / \varepsilon)$, on the other hand, then the reaction is made faster and faster as $\varepsilon \rightarrow 0$, resulting in a limit system in which the chemical reaction $A \leftrightharpoons B$ is in continuous equilibrium. Because of this, both $A$ and $B$ have the same concentration $u$, and $u$ solves the diffusion problem

$$
\begin{array}{ll}
\partial_{t} u=\Delta u, & \text { for } x \in \Omega, t>0 \\
u(0, x)=\frac{1}{2}\left(u^{0,+}(x)+u^{0,-}(x)\right) & \text { for } x \in \Omega .
\end{array}
$$

Note the instantaneous equilibration of the initial data in this system.

While the scaling in terms of $\varepsilon$ of $\tau_{\varepsilon}$ can not be chosen differently without obtaining structurally different limit systems, there is still a choice in the prefactor. For $\tau_{\varepsilon}:=\tilde{\tau} \varepsilon e^{1 / \varepsilon}$ with $\tilde{\tau}>0$ fixed, the prefactor $\tilde{\tau}$ will appear in the definition $\sqrt{15}$ of $k$.

There is a also a modelling aspect to the choice of $\tau$. In this paper we use no knowledge about the value of $\tau$ in the diffusion system at finite $\varepsilon$; the choice $\tau=\tau_{\varepsilon}$ is motivated by the wish to have a limit system that contains both diffusive and reactive terms. If one has additional information about the mobility of the system in the $x$ - and $\xi$-directions, then the value of $\tau$ will follow from this. 
Equal rate constants. The assumption of equal depth of the two minima of $H$ corresponds to the assumption (or, depending on one's point of view, the result) that the rate constant $k$ in (14) is the same for the two reactions $A \rightarrow B$ and $B \rightarrow A$. The general case requires a slightly different choice for $H$, as follows.

Let the original macroscopic equations for the evolution of $A$ and $B$ (in terms of densities that we also denote $A$ and $B$ ) be

$$
\begin{aligned}
\partial_{t} A-\Delta A & =k^{-} B-k^{+} A \\
\partial_{t} B-\Delta B & =k^{+} A-k^{-} B .
\end{aligned}
$$

Choose a fixed function $H_{0} \in C^{\infty}([-1,1])$ such that $H_{0}^{\prime}( \pm 1)=0$ and $H_{0}(1)-H_{0}(-1)=$ $\log k^{-}-\log k^{+}$. We then construct the enthalpy $H_{\varepsilon}$ by setting

$$
H_{\varepsilon}:=H_{0}+\frac{1}{\varepsilon} H,
$$

where $H$ is the same enthalpy function as above. The same proof as for the equal-well case then gives convergence of the finite- $\varepsilon$ problems to (27).

Equal diffusion constants. It is possible to change the setup such that the limiting system has different diffusion rate in $A$ and $B$. We first write equation 6 as

$$
\partial_{t} \rho-\operatorname{div} D_{\varepsilon} F_{\varepsilon}=0,
$$

where the mobility matrix $D_{\varepsilon} \in \mathbb{R}^{(d+1) \times(d+1)}$ and the flux $F_{\varepsilon}$ are given by

$$
D_{\varepsilon}=\left(\begin{array}{cc}
\mathrm{I} & 0 \\
0 & \tau_{\varepsilon}
\end{array}\right) \quad \text { and } \quad F_{\varepsilon}=F_{\varepsilon}(\rho)=\left(\begin{array}{c}
\nabla u \\
\nabla \rho+\rho \nabla H
\end{array}\right)
$$

By replacing the identity matrix block $I$ in $D_{\varepsilon}$ by a block of the form $a(\xi)$ I the $x$-directional diffusion can be modified as a function of $\xi$. This translates into two different diffusion coefficients for $A$ and $B$.

The function $H$. The limit result of Theorem 1 shows that only a small amount of information about the function $H$ propagates into the limit problem: specifically, the local second-order structure of $H$ around the wells and around the mountain-pass point.

One other aspect of the structure of $H$ is hidden: the fact that we rescaled the $\xi$ variable by a factor of $\sqrt{\tau_{\varepsilon}}$ can also be interpreted as a property of $H$, since the effective distance between the two wells, as measured against the intrinsic distance associated with the Brownian motion, is equal to $2 \sqrt{\tau_{\varepsilon}}$ after rescaling.

We also assumed in this paper that $H$ has only 'half' wells, in the sense that $H$ is defined on $[-1,1]$ instead of $\mathbb{R}$. This was for practical convenience, and one can do essentially the same analysis for a function $H$ that is defined on $\mathbb{R}$. In this case one will regain a slightly different value of $k$, namely $k=\sqrt{\left|H^{\prime \prime}(0)\right| H^{\prime \prime}(1)} / 2 \pi$. (For this reason this is also the value found by Kramers [12, equation (17)]).

Single particles versus multiple particles, and concentrations versus probabilities. The description of this paper of the system in terms of a probability measure $\rho$ on $D$ is the description of the probability of a single particle. This implies that the limit object $\left(u^{-}, u^{+}\right)$ should be interpreted as the density (with respect to $\gamma$ ) of a limiting probability measure, again describing a single particle.

This is at odds with common continuum modelling philosophy, where the main objects are concentrations (mass or volume) that represent a large number of particles; in this philosophy the solution $\left(u^{-}, u^{+}\right)$of 14 should be viewed as such a concentration, which is to say as the projection onto $x$-space of a joint probability distribution of a large number of particles.

For the simple reaction $A \leftrightharpoons B$ these two interpretations are actually equivalent. This arises from the fact that $A \rightarrow B$ reaction events in each of the particles are independent of each other; therefore the joint distribution of a large number $N$ of particles factorizes into a product of $N$ copies of the distribution of a single particle. For the case of this paper, therefore, the distinction between these two views is not important.

More general reactions. The remark above implies that the situation will be different for systems where reaction events cause differences in distributions between the particles, such 
as the reaction $A+B \leftrightarrows C$. This can be recognized as follows: a particle $A$ that has just separated from a $B$ particle (in a reaction event of the form $C \rightarrow A+B$ ) has a position that is highly correlated with the corresponding $B$ particle, while this is not the case for all the other $A$ particles. Therefore the $A$ particles will not have the same distribution. The best one can hope for is that in the limit of a large number of particles the distribution becomes the same in some weak way. This is one of the major obstacles in developing a similar connection as in this paper for more complex reaction equations.

\subsection{Plan of the paper}

One of the main difficulties in the proof of Theorem 1, namely the singular behaviour given by the concentration of the invariant measure $\gamma_{\varepsilon}$ onto the two lines at $\xi= \pm 1$, can be overcome by working in the underlying space of (signed or probability) measures in $\bar{D}$. This point of view is introduced in Section 2 Section 3 contains the basic $\Gamma$-convergence results (Theorem 4 and the proof of Theorem 1 and of Theorem 2 The argument showing the link between $\Gamma$ convergence of the quadratic forms $a_{\varepsilon}, b_{\varepsilon}$ and the convergence of the solutions to the evolution problems (see the comments in section 1.9) is presented in Section 4 in a general form, which can can be easily applied to other situations.

\section{Formulation of the evolution equations in measure spaces}

\section{The Kramers-Smoluchowski equation}

We first summarize the functional framework introduced above. Let us denote by $(\cdot, \cdot)_{\varepsilon}$ the scalar product in $\mathbb{R}^{d} \times \mathbb{R}$ defined by

$$
(\boldsymbol{x}, \boldsymbol{y})_{\varepsilon}:=x \cdot y+\tau_{\varepsilon} \xi \eta, \quad \text { for every } \boldsymbol{x}=(x, \xi), \boldsymbol{y}=(y, \eta) \in \mathbb{R}^{d} \times \mathbb{R},
$$

with the corresponding norm $\|\cdot\|_{\varepsilon}$. We introduced two Hilbert spaces

$$
H_{\varepsilon}:=L^{2}\left(D, \gamma_{\varepsilon}\right) \quad \text { and } \quad V_{\varepsilon}=W^{1,2}\left(D, \gamma_{\varepsilon}\right)
$$

and the bilinear forms

$$
\begin{array}{ll}
b_{\varepsilon}(u, v):=\int_{D} u v \mathrm{~d} \gamma_{\varepsilon} & \text { for every } u, v \in H_{\varepsilon}, \\
a_{\varepsilon}(u, v):=\int_{D}\left(\nabla_{x, \xi} u, \nabla_{x, \xi} v\right)_{\varepsilon} \mathrm{d} \gamma_{\varepsilon} & \text { for every } u, v \in V_{\varepsilon},
\end{array}
$$

with which 77 has the variational formulation

$$
b_{\varepsilon}\left(\partial_{t} u_{\varepsilon}, v\right)+a_{\varepsilon}\left(u_{\varepsilon}, v\right)=0 \quad \text { for every } v \in V_{\varepsilon}, t>0 ; \quad u_{\varepsilon}(0, \cdot)=u_{\varepsilon}^{0} .
$$

The main technical difficulty in studying the limit behaviour of 31 as $\varepsilon \downarrow 0$ consists of the $\varepsilon$-dependence of the functional spaces $H_{\varepsilon}, V_{\varepsilon}$. Since for our approach it is crucial to work in a fixed ambient space, we embed the solutions of (31) in the space of finite Borel measures $\mathscr{M}(\bar{D})$ by associating to $u_{\varepsilon}$ the measure $\rho_{\varepsilon}:=u_{\varepsilon} \gamma_{\varepsilon}$. We thus introduce the quadratic forms

$$
\begin{array}{ll}
\mathfrak{b}_{\varepsilon}(\rho):=b_{\varepsilon}(u, u) & \text { if } \rho \ll \gamma_{\varepsilon} \text { and } u=\frac{\mathrm{d} \rho}{\mathrm{d} \gamma_{\varepsilon}} \in H_{\varepsilon}, \\
\mathfrak{a}_{\varepsilon}(\rho):=a_{\varepsilon}(u, u) & \text { if } \rho \ll \gamma_{\varepsilon} \text { and } u=\frac{\mathrm{d} \rho}{\mathrm{d} \gamma_{\varepsilon}} \in V_{\varepsilon},
\end{array}
$$

trivially extended to $+\infty$ when $\rho$ is not absolutely continuous with respect to $\gamma_{\varepsilon}$ or its density $u$ does not belong to $H_{\varepsilon}$ or $V_{\varepsilon}$ respectively. Denoting by $\operatorname{Dom}\left(\mathfrak{a}_{\varepsilon}\right)$ and $\operatorname{Dom}\left(\mathfrak{b}_{\varepsilon}\right)$ their proper domains, we still denote by $\mathfrak{a}_{\varepsilon}(\cdot, \cdot)$ and $\mathfrak{b}_{\varepsilon}(\cdot, \cdot)$ the corresponding bilinear forms defined on $\operatorname{Dom}\left(\mathfrak{a}_{\varepsilon}\right)$ and $\operatorname{Dom}\left(\mathfrak{b}_{\varepsilon}\right)$ respectively. Setting $\rho_{\varepsilon}:=u_{\varepsilon} \gamma_{\varepsilon}, \sigma:=v \gamma_{\varepsilon}$, 31 is equivalent to the integrated form

$$
\mathfrak{b}_{\varepsilon}\left(\rho_{\varepsilon}(t), \sigma\right)+\int_{0}^{t} \mathfrak{a}_{\varepsilon}\left(\rho_{\varepsilon}(r), \sigma\right) \mathrm{d} r=\mathfrak{b}_{\varepsilon}\left(\rho_{\varepsilon}^{0}, \sigma\right) \quad \text { for every } \sigma \in \operatorname{Dom}\left(\mathfrak{a}_{\varepsilon}\right) .
$$


We also recall the standard estimates

$$
\begin{array}{ll}
\frac{1}{2} \mathfrak{b}_{\varepsilon}\left(\rho_{\varepsilon}(t)\right)+\int_{0}^{t} \mathfrak{a}_{\varepsilon}\left(\rho_{\varepsilon}(r)\right) \mathrm{d} r=\frac{1}{2} \mathfrak{b}_{\varepsilon}\left(\rho_{\varepsilon}^{0}\right) & \text { for every } t \geq 0 \\
t \mathfrak{a}_{\varepsilon}\left(\rho_{\varepsilon}(t)\right)+2 \int_{0}^{t} r \mathfrak{b}_{\varepsilon}\left(\partial_{t} \rho_{\varepsilon}(r)\right) \mathrm{d} r=\int_{0}^{t} \mathfrak{a}_{\varepsilon}\left(\rho_{\varepsilon}(r)\right) \mathrm{d} r & \text { for every } t \geq 0 \\
\frac{1}{2} \mathfrak{b}_{\varepsilon}\left(\rho_{\varepsilon}(t)\right)+t \mathfrak{a}_{\varepsilon}\left(\rho_{\varepsilon}(t)\right)+t^{2} \mathfrak{b}_{\varepsilon}\left(\partial_{t} \rho_{\varepsilon}(t)\right) \leq \frac{1}{2} \mathfrak{b}_{\varepsilon}\left(\rho_{\varepsilon}^{0}\right) & \text { for every } t>0
\end{array}
$$

Although versions of these expressions appear in various places, we were unable to find a reference that completely suits our purposes. We therefore briefly describe their proof, and we use the more conventional formulation in terms of the bilinear forms $a_{\varepsilon}$ and $b_{\varepsilon}$ and spaces $H_{\varepsilon}$ and $V_{\varepsilon}$; note that $b_{\varepsilon}$ is an inner product for $H_{\varepsilon}$, and $b_{\varepsilon}+a_{\varepsilon}$ is an inner product for $V_{\varepsilon}$.

When $u_{0}$ is sufficiently smooth, standard results (e.g. 6, Chapter VII]) provide the existence of a solution $u_{\varepsilon} \in C\left([0, \infty) ; V_{\varepsilon}\right) \cap C^{\infty}\left((0, \infty) ; V_{\varepsilon}\right)$, such that the functions $t \mapsto a_{\varepsilon}\left(u_{\varepsilon}(t)\right)$ and $t \mapsto b_{\varepsilon}\left(\partial_{t} u_{\varepsilon}(t)\right)$ are non-increasing; in addition, the solution operator (semigroup) $S_{t}$ is a contraction in $H_{\varepsilon}$. For this case all three expressions can be proved by differentiation.

In order to extend them to all $u_{\varepsilon}^{0} \in H_{\varepsilon}$, we note that for fixed $t>0$ the two norms on $H_{\varepsilon}$ given by (the square roots of)

$$
u_{\varepsilon}^{0} \mapsto \frac{1}{2} b_{\varepsilon}\left(u_{\varepsilon}^{0}\right) \quad \text { and } \quad u_{\varepsilon}^{0} \mapsto \frac{1}{2} b_{\varepsilon}\left(S_{t} u_{\varepsilon}^{0}\right)+\int_{0}^{t} a_{\varepsilon}\left(S_{r} u_{\varepsilon}^{0}\right) \mathrm{d} r
$$

are identical by (35) on a $H_{\varepsilon}$-dense subset. If we approximate a general $u_{\varepsilon}^{0} \in H_{\varepsilon}$ by smooth $u_{\varepsilon, n}^{0}$, then the sequence $u_{\varepsilon, n}^{0}$ is a Cauchy sequence with respect to both norms; by copying the proof of completeness of the space $L^{2}\left(0, \infty ; V_{\varepsilon}\right)$ (see e.g. 6, Th. IV.8]) it follows that the integral in 38 converges. This allows us to pass to the limit in 35 . The argument is similar for $(37)$, when one writes the sum of $(35)$ and $(36)$ as

$$
\frac{1}{2} b_{\varepsilon}\left(u_{\varepsilon}(t)\right)+t a_{\varepsilon}\left(u_{\varepsilon}(t)\right)+2 \int_{0}^{t} r b_{\varepsilon}\left(\partial_{t} u_{\varepsilon}(r)\right) \mathrm{d} r=\frac{1}{2} b_{\varepsilon}\left(u_{\varepsilon}^{0}\right) .
$$

Finally, 37 follows by 39 since $r \mapsto \mathfrak{b}_{\varepsilon}\left(\partial_{t} u_{\varepsilon}(r)\right)$ is non-increasing.

\section{The reaction-diffusion limit}

We now adopt the same point of view to formulate the limit reaction-diffusion system in the setting of measures. Recall that for $u \in H:=L^{2}(\bar{D}, \gamma)$ we set $u^{ \pm}(x):=u(x, \pm 1)$, and thus we defined the function space

$$
V:=\left\{u \in H: u^{ \pm} \in W^{1,2}(\Omega)\right\},
$$

and the bilinear forms

$$
\begin{aligned}
& b(u, v)=\frac{1}{2} \int_{\Omega}\left(u^{+} v^{+}+u^{-} v^{-}\right) \mathrm{d} \lambda_{\Omega}, \\
& a(u, v):=\frac{1}{2} \int_{\Omega}\left(\nabla_{x} u^{+} \nabla_{x} v^{+}+\nabla_{x} u^{-} \nabla_{x} v^{-}+k\left(u^{+}-u^{-}\right)\left(v^{+}-v^{-}\right)\right) \mathrm{d} \lambda_{\Omega} .
\end{aligned}
$$

As before we now extend these definitions to arbitrary measures by

$$
\begin{array}{ll}
\mathfrak{b}(\rho):=b(u, u) & \text { if } \rho \ll \gamma \text { and } u=\frac{\mathrm{d} \rho}{\mathrm{d} \gamma} \in H, \\
\mathfrak{a}(\rho):=a(u, u) & \text { if } \rho \ll \gamma \text { and } u=\frac{\mathrm{d} \rho}{\mathrm{d} \gamma} \in V,
\end{array}
$$

with corresponding bilinear forms $\mathfrak{b}(\cdot, \cdot)$ and $\mathfrak{a}(\cdot, \cdot)$; problem $14 \mathrm{a}, \mathrm{b}, \mathrm{c})$ can be reformulated as

$$
\mathfrak{b}\left(\partial_{t} \rho(t), \sigma\right)+\mathfrak{a}(\rho(t), \sigma)=0 \quad \text { for every } t>0 \text { and } \sigma \in \operatorname{Dom}(a),
$$

or in the integral form

$$
\mathfrak{b}(\rho(t), \sigma)+\int_{0}^{t} \mathfrak{a}(\rho(r), \sigma) \mathrm{d} r=\mathfrak{b}\left(\rho^{0}, \sigma\right) \quad \text { for every } \sigma \in \operatorname{Dom}(\mathfrak{a}) .
$$

Since both problems (34) and 44 are embedded in the same measure space $\mathscr{M}(\bar{D})$, we can study the convergence of the solution $\rho_{\varepsilon}$ of $(34)$ as $\varepsilon \downarrow 0$. 


\section{$3 \Gamma$-convergence result for the quadratic forms $\mathfrak{a}_{\varepsilon}, \mathfrak{b}_{\varepsilon}$}

The aim of this section is to prove the following $\Gamma$-convergence result:

Theorem 4. If $\rho_{\varepsilon} \rightarrow \rho$ as $\varepsilon \downarrow 0$ in $\mathscr{M}(\bar{D})$ then

$$
\liminf _{\varepsilon \downarrow 0} \mathfrak{a}_{\varepsilon}\left(\rho_{\varepsilon}\right) \geq \mathfrak{a}(\rho), \quad \liminf _{\varepsilon \downarrow 0} \mathfrak{b}_{\varepsilon}\left(\rho_{\varepsilon}\right) \geq \mathfrak{b}(\rho) .
$$

For every $\rho \in \mathscr{M}(\bar{D})$ such that $\mathfrak{a}(\rho)+\mathfrak{b}(\rho)<+\infty$ there exists a family $\rho_{\varepsilon} \in \mathscr{M}(\bar{D})$ weakly-* converging to $\rho$ such that

$$
\lim _{\varepsilon \downarrow 0} \mathfrak{a}_{\varepsilon}\left(\rho_{\varepsilon}\right)=\mathfrak{a}(\rho), \quad \lim _{\varepsilon \downarrow 0} \mathfrak{b}_{\varepsilon}\left(\rho_{\varepsilon}\right)=\mathfrak{b}(\rho)
$$

Note that $\mathscr{M}(\bar{D})$ endowed with the weak-* topology is the dual of a separable Banach space, and therefore the sequential definition of $\Gamma$-convergence coincides with the topological definition 8, Proposition 8.1 and Theorem 8.10]; consequently Theorem 4 implies the $\Gamma$ convergence of the families $\mathfrak{a}_{\varepsilon}$ and $\mathfrak{b}_{\varepsilon}$. Theorem 4 actually states a stronger result, since the recovery sequence can be chosen to be the same for $\mathfrak{a}_{\varepsilon}$ and $\mathfrak{b}_{\varepsilon}$. This joint $\Gamma$-convergence of the families $\mathfrak{a}_{\varepsilon}$ and $\mathfrak{b}_{\varepsilon}$ is nearly equivalent with $\Gamma$-convergence of combined quadratic forms:

Lemma 5. Theorem 4 implies the

$$
\Gamma(\mathscr{M}(\bar{D})) \text {-convergence of } \quad \mathfrak{q}_{\varepsilon}^{\kappa}(\rho):=\mathfrak{b}_{\varepsilon}(\rho)+\kappa \mathfrak{a}_{\varepsilon}(\rho) \quad \text { to } \quad \mathfrak{q}^{\kappa}(\rho):=\mathfrak{b}(\rho)+\kappa \mathfrak{a}(\rho)
$$

for each $\kappa>0$.

Conversely, if we assume (47), then (46) holds, and (45) follows under the additional assumption

$$
\limsup _{\varepsilon \downarrow 0} \mathfrak{a}_{\varepsilon}\left(\rho_{\varepsilon}\right)+\mathfrak{b}_{\varepsilon}\left(\rho_{\varepsilon}\right)=C<+\infty .
$$

Proof. The first part of the Lemma is immediate. For the second part, suppose that $\rho_{\varepsilon} \rightarrow \rho$ and satisfies 48 ; the $\Gamma$-liminf inequality for $\mathfrak{q}_{\varepsilon}^{\kappa}$ yields

$$
\liminf _{\varepsilon \downarrow 0} \mathfrak{b}_{\varepsilon}\left(\rho_{\varepsilon}\right) \geq \liminf _{\varepsilon \downarrow 0} \mathfrak{q}_{\varepsilon}^{k}\left(\rho_{\varepsilon}\right)-C \kappa \geq \mathfrak{q}^{\kappa}(\rho)-C \kappa=\mathfrak{b}(\rho)+\kappa(\mathfrak{a}(\rho)-C) \quad \text { for every } \kappa>0,
$$

and therefore the second inequality of 45 follows by letting $\kappa \downarrow 0$. A similar argument yields the first inequality of 45 .

Concerning 46, $\Gamma$-convergence of $\mathfrak{q}_{\varepsilon}^{1}$ to $\mathfrak{q}^{1}$ yields a recovery family $\rho_{\varepsilon} \rightarrow \rho$ such that

$$
\lim _{\varepsilon \downarrow 0} \mathfrak{a}_{\varepsilon}\left(\rho_{\varepsilon}\right)+\mathfrak{b}_{\varepsilon}\left(\rho_{\varepsilon}\right)=\mathfrak{a}(\rho)+\mathfrak{b}(\rho)<+\infty ;
$$

In particular $\mathfrak{a}_{\varepsilon}\left(\rho_{\varepsilon}\right)+\mathfrak{b}_{\varepsilon}\left(\rho_{\varepsilon}\right)$ is uniformly bounded, so that 45 yields the separate convergence 46.

One of the most useful consequences of 47) is contained in the next result (see e.g. 16 Lemma 3.6]).

Corollary 6 (Weak-strong convergence). Assume that (47) holds for every $\kappa>0$ and let $\rho_{\varepsilon}, \sigma_{\varepsilon} \in \mathscr{M}(\bar{D})$ be two families weakly converging to $\rho, \sigma$ as $\varepsilon \downarrow 0$ and satisfying the uniform bound 48 , i.e.

$$
\underset{\varepsilon \downarrow 0}{\limsup } \mathfrak{a}_{\varepsilon}\left(\rho_{\varepsilon}\right)+\mathfrak{b}_{\varepsilon}\left(\rho_{\varepsilon}\right)<+\infty, \quad \limsup _{\varepsilon \downarrow 0} \mathfrak{a}_{\varepsilon}\left(\sigma_{\varepsilon}\right)+\mathfrak{b}_{\varepsilon}\left(\sigma_{\varepsilon}\right)<+\infty,
$$

so that $\rho, \sigma$ belong to the domains of the bilinear form $\mathfrak{a}$ and $\mathfrak{b}$. We have

$$
\begin{array}{lll}
\lim _{\varepsilon \downarrow 0} \mathfrak{a}_{\varepsilon}\left(\sigma_{\varepsilon}\right)=\mathfrak{a}(\sigma) & \Longrightarrow & \lim _{\varepsilon \downarrow 0} \mathfrak{a}_{\varepsilon}\left(\rho_{\varepsilon}, \sigma_{\varepsilon}\right)=\mathfrak{a}(\rho, \sigma) \\
\lim _{\varepsilon \downarrow 0} \mathfrak{b}_{\varepsilon}\left(\sigma_{\varepsilon}\right)=\mathfrak{b}(\sigma) & \Longrightarrow & \lim _{\varepsilon \downarrow 0} \mathfrak{b}_{\varepsilon}\left(\rho_{\varepsilon}, \sigma_{\varepsilon}\right)=\mathfrak{b}(\rho, \sigma) .
\end{array}
$$


Proof. We reproduce here the proof of [16] in the case of the quadratic forms $\mathfrak{a}_{\varepsilon}$ (50). Note that by (49) and Lemma 5 we can assume that $\rho_{\varepsilon}$ and $\sigma_{\varepsilon}$ satisfy (45. For every positive scalar $r>0$ we have

$$
2 \mathfrak{a}_{\varepsilon}\left(\rho_{\varepsilon}, \sigma_{\varepsilon}\right)=2 \mathfrak{a}_{\varepsilon}\left(r \rho_{\varepsilon}, r^{-1} \sigma_{\varepsilon}\right)=\mathfrak{a}_{\varepsilon}\left(r \rho_{\varepsilon}+r^{-1} \sigma_{\varepsilon}\right)-r^{2} \mathfrak{a}_{\varepsilon}\left(\rho_{\varepsilon}\right)-r^{-2} \mathfrak{a}_{\varepsilon}\left(\sigma_{\varepsilon}\right) .
$$

Taking the inferior limit as $\varepsilon \downarrow 0$ and recalling 45 we get for $A:=\lim \sup _{\varepsilon \downarrow 0} \mathfrak{a}_{\varepsilon}\left(\rho_{\varepsilon}\right)$

$$
\liminf _{\varepsilon \downarrow 0} 2 \mathfrak{a}_{\varepsilon}\left(\rho_{\varepsilon}, \sigma_{\varepsilon}\right) \geq \mathfrak{a}\left(r \rho+r^{-1} \sigma\right)-r^{2} A-r^{-2} \mathfrak{a}(\sigma)=2 \mathfrak{a}(\rho, \sigma)+r^{2}(\mathfrak{a}(\rho)-A) .
$$

Since $r>0$ is arbitrary and $A$ is finite by 49 we obtain $\lim \inf _{\varepsilon \downarrow} \mathfrak{a}_{\varepsilon}\left(\rho_{\varepsilon}, \sigma_{\varepsilon}\right) \geq \mathfrak{a}(\rho, \sigma)$ and inverting the sign of $\sigma$ we get $(50)$.

We split the proof of Theorem 4 in various steps.

\subsection{Estimates near $\Omega \times\{-1,1\}$.}

Lemma 7. If $\rho_{\varepsilon}=u_{\varepsilon} \gamma_{\varepsilon}$ satisfies the uniform bound $\mathfrak{a}_{\varepsilon}\left(\rho_{\varepsilon}\right) \leq C<+\infty$ for every $\varepsilon>0$, then for every $\delta \in(0,1)$

$$
\partial_{\xi} u_{\varepsilon} \rightarrow 0 \quad \text { in } L^{2}\left(\Omega \times \omega_{\delta}\right), \quad \text { as } \varepsilon \rightarrow 0,
$$

where $\omega_{\delta}:=(-1,-\delta) \cup(\delta, 1)$.

Proof. We observe that

$$
\tau_{\varepsilon} \int_{D}\left(\partial_{\xi} u_{\varepsilon}\right)^{2} \mathrm{~d} \gamma_{\varepsilon} \leq \mathfrak{a}_{\varepsilon}\left(\rho_{\varepsilon}\right) \leq C<\infty
$$

If $h_{\delta}=\sup _{\xi \in \omega_{\delta}} H(\xi)<1$, then $\inf _{\xi \in \omega_{\delta}} e^{-H(\xi) / \varepsilon}=e^{-h_{\delta} / \varepsilon}$, and we find

$$
\int_{\Omega \times \omega_{\delta}}\left(\partial_{\xi} u_{\varepsilon}\right)^{2} \mathrm{~d} x \mathrm{~d} \xi \leq C \frac{Z_{\varepsilon}}{\tau_{\varepsilon}} e^{\frac{h_{\delta}}{\varepsilon}}=C \frac{Z_{\varepsilon}}{\varepsilon} e^{\frac{h_{\delta}-1}{\varepsilon}} .
$$

Taking the limit as $\varepsilon \rightarrow 0$ we obtain 52 .

Lemma 8 (Convergence of traces). Let us suppose that $\rho_{\varepsilon}=u_{\varepsilon} \gamma_{\varepsilon} \rightarrow \rho=u \gamma$ with $\mathfrak{a}_{\varepsilon}\left(\rho_{\varepsilon}\right) \leq$ $C<+\infty$ and let $u_{\varepsilon}^{ \pm}(x)$ be the traces of $u_{\varepsilon}$ at $\xi= \pm 1$. Then as $\varepsilon \downarrow 0$

$$
u_{\varepsilon}^{ \pm} \rightarrow u^{ \pm} \text {strongly in } L^{2}(\Omega)
$$

where $u^{ \pm}$are the functions given by 13 .

Proof. Let us consider, e.g., the case of $u_{\varepsilon}^{-}$. Let us fix $\delta \in(0,1)$; by 52 and standard trace results in $W^{1,2}(-1,-1+\delta)$ we know that

$\lim _{\varepsilon \downarrow 0} \int_{\Omega} \omega_{\varepsilon}^{2}(x) d \mathcal{L}^{d}=0 \quad$ where $\quad \omega_{\varepsilon}^{2}(x):=\sup _{-1 \leq \xi \leq-1+\delta}\left|u_{\varepsilon}(x, \xi)-u_{\varepsilon}^{-}(x)\right|^{2} \leq \delta \int_{-1}^{-1+\delta}\left|\partial_{\xi} u_{\varepsilon}(x, \xi)\right|^{2} \mathrm{~d} \xi$.

Let us fix a function $\phi \in C^{0}(\Omega)$ and a function $\psi \in C^{0}[-1,1]$ with $0 \leq \psi \leq 1, \psi(-1)=1$, $\operatorname{supp} \psi \subset[-1,-1+\delta]$; we set

$$
J_{\varepsilon}:=\int_{-1}^{1} \psi(\xi) \mathrm{d} \tilde{\gamma}_{\varepsilon}(\xi), \quad \tilde{u}_{\varepsilon}(x):=J_{\varepsilon}^{-1} \int_{-1}^{1} u_{\varepsilon}(x, \xi) \psi(\xi) d \tilde{\gamma}_{\varepsilon}(\xi),
$$

where $\tilde{\gamma}_{\varepsilon}$ is the measure defined in $(3)$. Note that

$$
\lim _{\varepsilon \rightarrow 0} J_{\varepsilon}=\langle\psi, \gamma\rangle=\frac{1}{2} \psi(-1)+\frac{1}{2} \psi(1)=\frac{1}{2} .
$$

Since $\rho_{\varepsilon}$ weakly converge to $\rho$ we know that

$$
\lim _{\varepsilon \downarrow 0} \int_{\Omega} \phi(x) \tilde{u}_{\varepsilon}(x) \mathrm{d} \lambda_{\Omega}=\lim _{\varepsilon \downarrow 0} J_{\varepsilon}^{-1} \int_{\Omega} \phi(x) \psi(\xi) u_{\varepsilon}(x, \xi) \mathrm{d} \gamma_{\varepsilon}(x, \xi)=\int_{\Omega} \phi(x) u^{-}(x) \mathrm{d} \lambda_{\Omega}
$$


so that $\tilde{u}_{\varepsilon}$ converges to $u^{-}$in the duality with bounded continuous functions. On the other hand,

$$
\int_{\Omega}\left|\nabla_{x} \tilde{u}_{\varepsilon}(x)\right|^{2} \mathrm{~d} \lambda_{\Omega} \leq J_{\varepsilon}^{-1} \int_{\Omega} \int_{-1}^{1}\left|\nabla_{x} u_{\varepsilon}(x, \xi)\right|^{2} \psi(\xi) \mathrm{d} \tilde{\gamma}(\xi) \mathrm{d} \lambda_{\Omega}(x) \leq J_{\varepsilon}^{-1} \mathfrak{a}_{\varepsilon}\left(\rho_{\varepsilon}\right) \leq 2 C
$$

so that $\tilde{u}_{\varepsilon} \rightarrow u^{-}$in $L^{2}(\Omega)$ by Rellich compactness theorem.

On the other hand, thanks to 54, we have

$$
\begin{aligned}
& \lim _{\varepsilon \downarrow 0} \int_{\Omega}\left|u_{\varepsilon}^{-}(x)-\tilde{u}_{\varepsilon}(x)\right|^{2} \mathrm{~d} \lambda_{\Omega}(x)=\lim _{\varepsilon \downarrow 0} J_{\varepsilon}^{-2} \int_{\Omega}\left|\int_{-1}^{1} \psi(\xi)\left(u_{\varepsilon}(x, \xi)-u^{-}(x)\right) \mathrm{d} \tilde{\gamma}_{\varepsilon}(\xi)\right|^{2} \mathrm{~d} \lambda_{\Omega}(x) \\
& \quad \leq \lim _{\varepsilon \downarrow 0} \int_{D} \psi(\xi) \omega_{\varepsilon}^{2}(x) \mathrm{d} \gamma_{\varepsilon}(x, \xi)=0,
\end{aligned}
$$

which yields 53 .

Remark. A completely analogous argument shows that if $\rho_{\varepsilon}$ satisfies a $W^{1,1}\left(D ; \gamma_{\varepsilon}\right)$-uniform bound

$$
\int_{D}\left\|\nabla_{x, \xi} u_{\varepsilon}\right\|_{\varepsilon} \mathrm{d} \gamma_{\varepsilon}(x, \xi) \leq C<+\infty
$$

instead of $\mathfrak{a}_{\varepsilon}\left(\rho_{\varepsilon}\right) \leq C$, then $u_{\varepsilon}^{ \pm} \rightarrow u^{ \pm}$in $L^{1}(\Omega)$.

\subsection{Asymptotics for the minimal transition cost.}

Given $\left(\varphi^{-}, \varphi^{+}\right) \in \mathbb{R}^{2}$ let us set

$$
K_{\varepsilon}\left(\varphi^{-}, \varphi^{+}\right):=\min \left\{\tau_{\varepsilon} \int_{-1}^{1}\left(\varphi^{\prime}(\xi)\right)^{2} d \tilde{\gamma}_{\varepsilon}: \varphi \in W^{1,2}(-1,1), \varphi( \pm 1)=\varphi^{ \pm}\right\}
$$

It is immediate to check that $K_{\varepsilon}$ is a quadratic form depending only on $\varphi^{+}-\varphi^{-}$, i.e.

$$
K_{\varepsilon}\left(\varphi^{-}, \varphi^{+}\right)=k_{\varepsilon}\left(\varphi^{+}-\varphi^{-}\right)^{2}, \quad k_{\varepsilon}=K_{\varepsilon}(-1 / 2,1 / 2) .
$$

We call $\mathcal{T}_{\varepsilon}\left(\varphi^{-}, \varphi^{+}\right)$the solution of the minimum problem $(56)$ : it admits the simple representation

$$
\mathcal{T}_{\varepsilon}\left(\varphi^{-}, \varphi^{+}\right)=\frac{1}{2}\left(\varphi^{-}+\varphi^{+}\right)+\left(\varphi^{+}-\varphi^{-}\right) \phi_{\varepsilon}
$$

where $\phi_{\varepsilon}=\mathcal{T}_{\varepsilon}(-1 / 2,1 / 2)$. We also set

$$
Q_{\varepsilon}\left(\varphi^{-}, \varphi^{+}\right):=\int_{-1}^{1}\left(\mathcal{T}_{\varepsilon}\left(\varphi^{-}, \varphi^{+}\right)\right)^{2} \mathrm{~d} \tilde{\gamma}_{\varepsilon}=\frac{1}{2}\left(\left(\varphi^{-}\right)^{2}+\left(\varphi^{+}\right)^{2}\right)+\left(q_{\varepsilon}-\frac{1}{4}\right)\left(\varphi^{+}-\varphi^{-}\right)^{2}
$$

where

$$
q_{\varepsilon}:=\int_{-1}^{1}\left|\phi_{\varepsilon}(\xi)\right|^{2} \mathrm{~d} \tilde{\gamma}_{\varepsilon}(\xi)=Q_{\varepsilon}(-1 / 2,1 / 2)
$$

Lemma 9. We have

$$
\lim _{\varepsilon \downarrow 0} k_{\varepsilon}=\frac{k}{2}=\frac{\sqrt{-H^{\prime \prime}(0) H^{\prime \prime}(1)}}{2 \pi}
$$

and

$$
\lim _{\varepsilon \downarrow 0} q_{\varepsilon}=\frac{1}{4} \quad \text { so that } \quad \lim _{\varepsilon \downarrow 0} Q_{\varepsilon}\left(\varphi^{-}, \varphi^{+}\right)=\frac{1}{2}\left(\varphi^{-}\right)^{2}+\frac{1}{2}\left(\varphi^{+}\right)^{2}
$$

Proof. $\phi_{\varepsilon}$ solves the Euler equation

$$
\left(e^{-H(\xi) / \varepsilon} \phi_{\varepsilon}^{\prime}(\xi)\right)^{\prime}=0 \quad \text { on }(-1,1), \quad \phi_{\varepsilon}( \pm 1)= \pm \frac{1}{2} .
$$

We can compute an explicit solution of 63 by integration:

$$
\phi_{\varepsilon}^{\prime}(\xi)=C e^{H(\xi) / \varepsilon}, \quad \phi_{\varepsilon}(\xi)=C^{\prime}+C \int_{0}^{\xi} e^{H(\eta) / \varepsilon} \mathrm{d} \eta .
$$


Define $I_{\varepsilon}:=\int_{-1}^{1} e^{H(\xi) / \varepsilon} \mathrm{d} \xi$. The boundary conditions for $\xi= \pm 1$ give

$$
C^{\prime}=0, \quad C \int_{-1}^{1} e^{H(\xi) / \varepsilon} d \xi=C I_{\varepsilon}=1
$$

It follows that

$$
\phi_{\varepsilon}(\xi)=I_{\varepsilon}^{-1} \int_{0}^{\xi} e^{H(\eta) / \varepsilon} \mathrm{d} \eta
$$

and

$$
k_{\varepsilon}=\tau_{\varepsilon} I_{\varepsilon}^{-2} \int_{-1}^{1} e^{2 H(\xi) / \varepsilon} \mathrm{d} \tilde{\gamma}_{\varepsilon}(\xi)=\tau_{\varepsilon} Z^{-1} I_{\varepsilon}^{-1} .
$$

We compute, using Laplace's method:

$$
I_{\varepsilon}=\sqrt{\frac{2 \pi \varepsilon}{\left|H^{\prime \prime}(0)\right|}} e^{1 / \varepsilon}(1+o(1)) \quad \text { and } \quad Z_{\varepsilon}=\sqrt{\frac{2 \pi \varepsilon}{H^{\prime \prime}(1)}}(1+o(1)), \quad \text { as } \varepsilon \rightarrow 0,
$$

thus obtaining 61. Since

$$
\phi_{\varepsilon}^{\prime}=I_{\varepsilon}^{-1} e^{H / \varepsilon} \rightarrow \delta_{0} \quad \text { in } \mathscr{D}^{\prime}(-1,1)
$$

and $H$ is even, we have

$$
\phi_{\varepsilon}(\xi)=I_{\varepsilon}^{-1} \int_{0}^{\xi} e^{H(\eta) / \varepsilon} \mathrm{d} \eta \rightarrow \frac{1}{2} \operatorname{sign}(\xi)
$$

uniformly on each compact subset of $[-1,1]$ not containing 0 . Since the range of $\phi_{\varepsilon}$ belongs to $[-1 / 2,1 / 2]$ and $\tilde{\gamma}_{\varepsilon} \rightarrow \frac{1}{2} \delta_{-1}+\frac{1}{2} \delta_{+1}$ we obtain 62 .

\subsection{End of the proof of Theorem 4 .}

The second limit of 45 follows by general lower semicontinuity results on integral functionals of measures, see e.g. [2, Lemma 9.4.3].

Concerning the first "lim inf" inequality, we split the the quadratic form $\mathfrak{a}_{\varepsilon}$ in the sum of two parts,

$$
\mathfrak{a}_{\varepsilon}^{1}\left(\rho_{\varepsilon}\right):=\int_{D}\left|\nabla_{x} u_{\varepsilon}(x, \xi)\right|^{2} \mathrm{~d} \gamma_{\varepsilon}(x, \xi), \quad \mathfrak{a}_{\varepsilon}^{2}\left(\rho_{\varepsilon}\right):=\tau_{\varepsilon} \int_{D}\left(\partial_{\xi} u_{\varepsilon}\right)^{2} \mathrm{~d} \gamma_{\varepsilon}(x, \xi) .
$$

We choose a smooth cutoff function $\eta^{-}:[-1,1] \rightarrow[0,1]$ such that $\eta^{-}(-1)=1$ and $\operatorname{supp}\left(\eta^{-}\right) \subset$ $[-1,-1 / 2]$ and the symmetric one $\eta^{+}(\xi):=\eta(-\xi)$. We also set

$$
u_{\varepsilon}^{-}(x):=\int_{-1}^{1} \eta^{-}(\xi) u_{\varepsilon}(x, \xi) \mathrm{d} \tilde{\gamma}_{\varepsilon}(\xi), \quad u_{\varepsilon}^{+}(x):=\int_{-1}^{1} \eta^{+}(\xi) u_{\varepsilon}(x, \xi) \mathrm{d} \tilde{\gamma}_{\varepsilon}(\xi)
$$

and it is easy to check that

$$
u_{\varepsilon}^{ \pm} \rightarrow \frac{1}{2} u^{ \pm} \quad \text { in } \mathscr{D}^{\prime}(\Omega)
$$

We also set $\theta_{\varepsilon}:=\int_{-1}^{1} \eta^{+}(\xi) \mathrm{d} \tilde{\gamma}_{\varepsilon}(\xi)\left(=\int_{-1}^{1} \eta^{-}(\xi) \mathrm{d} \tilde{\gamma}_{\varepsilon}(\xi)\right)$, observing that $\theta_{\varepsilon} \rightarrow 1 / 2$. We then have by Jensen inequality

$$
\mathfrak{a}_{\varepsilon}^{1}\left(\rho_{\varepsilon}\right) \geq \int_{\Omega} \int_{-1}^{1}\left(\eta^{-}(\xi)+\eta^{+}(\xi)\right)\left|\nabla_{x} u_{\varepsilon}\right|^{2} \mathrm{~d} \tilde{\gamma}_{\varepsilon}(\xi) \mathrm{d} \lambda_{\Omega} \geq \theta_{\varepsilon}^{-1} \int_{\Omega}\left|\nabla u_{\varepsilon}^{-}\right|^{2}+\left|\nabla u_{\varepsilon}^{+}\right|^{2} \mathrm{~d} \lambda_{\Omega}
$$

and, passing to the limit,

$$
\liminf _{\varepsilon \downarrow 0} \mathfrak{a}_{\varepsilon}^{1}\left(\rho_{\varepsilon}\right) \geq \frac{1}{2} \int_{\Omega}\left|\nabla u^{-}\right|^{2}+\left|\nabla u^{+}\right|^{2} \mathrm{~d} \lambda_{\Omega}
$$

Let us now consider the behaviour of $\mathfrak{a}_{\varepsilon}^{2}$ : applying (56) and 57) we get

$$
\mathfrak{a}_{\varepsilon}^{2}\left(\rho_{\varepsilon}\right)=\int_{\Omega}\left(\tau_{\varepsilon} \int_{-1}^{1}\left(\partial_{\xi} u_{\varepsilon}(x, \xi)\right)^{2} \mathrm{~d} \tilde{\gamma}_{\varepsilon}(\xi)\right) \mathrm{d} \lambda_{\Omega} \geq \int_{\Omega} k_{\varepsilon}\left(u_{\varepsilon}^{-}(x)-u_{\varepsilon}^{+}(x)\right)^{2} \mathrm{~d} \lambda_{\Omega}
$$


so that by 61 and 53 we obtain

$$
\liminf _{\varepsilon \downarrow 0} \mathfrak{a}_{\varepsilon}^{2}\left(\rho_{\varepsilon}\right) \geq \frac{k}{2} \int_{\Omega}\left(u^{-}(x)-u^{+}(x)\right)^{2} \mathrm{~d} \lambda_{\Omega} .
$$

In order to prove the "limsup" inequality (46) we fix $\rho=u \gamma$ with $u$ in the domain of the quadratic forms $a$ and $b$ so that $u^{ \pm}=u(\cdot, \pm 1)$ belong to $W^{1,2}(\Omega)$, and we set $\rho_{\varepsilon}=u_{\varepsilon} \gamma_{\varepsilon}$ where $u_{\varepsilon}(x, \cdot)=\mathcal{T}_{\varepsilon}\left(u^{-}(x), u^{+}(x)\right)$ as in (58). We easily have by 62 and the Lebesgue dominated convergence theorem

$$
\lim _{\varepsilon \downarrow 0} \mathfrak{b}_{\varepsilon}\left(\rho_{\varepsilon}\right)=\lim _{\varepsilon \downarrow 0} \int_{\Omega} Q_{\varepsilon}\left(u^{-}(x), u^{+}(x)\right) \mathrm{d} \lambda_{\Omega}=\int_{\Omega}\left(\frac{1}{2}\left|u^{-}(x)\right|^{2}+\frac{1}{2}\left|u^{+}(x)\right|^{2}\right) \mathrm{d} \lambda_{\Omega}=\mathfrak{b}(\rho) .
$$

Similarly, since for every $j=1, \cdots, d$ and almost every $x \in \Omega$

$$
\partial_{x_{j}} u_{\varepsilon}(x, \xi)=\mathcal{T}\left(\partial_{x_{j}} u^{-}(x), \partial_{x_{j}} u^{+}\right),
$$

we have

$$
\begin{aligned}
\lim _{\varepsilon \downarrow 0} \mathfrak{a}_{\varepsilon}\left(\rho_{\varepsilon}\right) & =\lim _{\varepsilon \downarrow 0} \int_{\Omega}\left(\sum_{j=1}^{d} Q_{\varepsilon}\left(\partial_{x_{j}} u^{-}(x), \partial_{x_{j}} u^{+}(x)\right)+K_{\varepsilon}\left(u^{-}(x), u^{+}(x)\right)\right) \mathrm{d} \lambda_{\Omega}= \\
& =\int_{\Omega}\left(\frac{1}{2}\left|\nabla u^{-}(x)\right|^{2}+\frac{1}{2}\left|\nabla u^{+}(x)\right|^{2}+\frac{k}{2}\left(u^{-}(x)-u^{+}(x)\right)\right) \mathrm{d} \lambda_{\Omega}=\mathfrak{a}(\rho) .
\end{aligned}
$$

\section{From $\Gamma$-convergence to convergence of the evolu- tion problems: proof of Theorems 1 and 2 .}

Having at our disposal the $\Gamma$-convergence result of Theorem 4 and its Corollary 6 it is not difficult to pass to the limit in the integrated equation (34).

Let us first notice that the quadratic forms $\mathfrak{b}_{\varepsilon}$ satisfy a uniform coercivity condition:

Lemma 10 (Uniform coercivity of $\mathfrak{b}_{\varepsilon}$ ). Every family of measures $\rho_{\varepsilon} \in \mathscr{M}(\bar{D}), \varepsilon>0$ satisfying

$$
\limsup _{\varepsilon>0} \mathfrak{b}_{\varepsilon}\left(\rho_{\varepsilon}\right)<+\infty
$$

is bounded in $\mathscr{M}(\bar{D})$ and admits a weakly-* converging subsequence.

Proof. It follows immediately by the fact that $\gamma_{\varepsilon}$ is a probability measure and therefore

$$
\left|\rho_{\varepsilon}\right|(\bar{D}) \leq\left(\mathfrak{b}_{\varepsilon}\left(\rho_{\varepsilon}\right)\right)^{1 / 2}
$$

(68) thus implies that the total mass of $\rho_{\varepsilon}$ is uniformly bounded and we can apply the relative weak-* compactness of bounded sets in dual Banach spaces.

The proof of Theorems 1 and 2 is a consequence of the following general result:

Theorem 11 (Convergence of evolution problems). Let us consider weakly-* lower-semicontinuous, nonnegative and extended-valued quadratic forms $\mathfrak{a}_{\varepsilon}, \mathfrak{b}_{\varepsilon}, \mathfrak{a}, \mathfrak{b}$ defined on $\mathscr{M}(\bar{D})$ and let us suppose that

1) Non degeneracy of the limit forms: $\mathfrak{b}$ is non degenerate (i.e. $\mathfrak{b}(\rho)=0 \Rightarrow \rho=0$ ) and $\operatorname{Dom}(\mathfrak{a})$ is dense in $\operatorname{Dom}(\mathfrak{b})$ with respect to the norm-convergence induced by $\mathfrak{b}$.

2) Uniform coercivity: $\mathfrak{b}_{\varepsilon}$ satisfy the coercivity property stated in the previous Lemma 10.

3) Joint $\Gamma$-convergence: $\mathfrak{q}_{\varepsilon}^{\kappa}:=\mathfrak{b}_{\varepsilon}+\kappa \mathfrak{a}_{\varepsilon}$ satisfy the joint $\Gamma$-convergence property 47

$$
\Gamma(\mathscr{M}(\bar{D}))-\lim _{\varepsilon \downarrow 0} \mathfrak{q}_{\varepsilon}^{\kappa}=\mathfrak{q}^{\kappa}=\mathfrak{b}+\kappa \mathfrak{a} \quad \text { for every } \kappa>0 .
$$


Let $\rho_{\varepsilon}(t), t \geq 0$, be the solution of the evolution problem (34) starting from $\rho_{\varepsilon}^{0} \in \operatorname{Dom}\left(\mathfrak{b}_{\varepsilon}\right)$. If

$$
\rho_{\varepsilon}^{0} \rightarrow \rho^{0} \text { in } \mathscr{M}(\bar{D}) \text { as } \varepsilon \downarrow 0 \quad \text { with } \quad \limsup _{\varepsilon \downarrow 0} \mathfrak{b}_{\varepsilon}\left(\rho_{\varepsilon}^{0}\right)<+\infty
$$

then $\rho_{\varepsilon}(t) \rightarrow \rho(t)$ in $\mathscr{M}(\bar{D})$ as $\varepsilon \downarrow 0$ for every $t>0$ and $\rho(t)$ is the solution of the limit evolution problem (44).

If moreover $\lim _{\mathcal{\varepsilon} \downarrow} \mathfrak{b}_{\varepsilon}\left(\rho_{\varepsilon}^{0}\right)=\mathfrak{b}\left(\rho_{0}\right)$ then

$$
\lim _{\varepsilon \downarrow 0} \mathfrak{b}_{\varepsilon}\left(\rho_{\varepsilon}(t)\right)=\mathfrak{b}(\rho(t)), \quad \lim _{\varepsilon \downarrow 0} \mathfrak{a}_{\varepsilon}\left(\rho_{\varepsilon}(t)\right)=\mathfrak{a}(\rho(t)) \quad \text { for every } t>0 .
$$

Proof. Let us first note that by 35 and the coercivity property of $\mathfrak{b}_{\varepsilon}$ the mass of $\rho_{\varepsilon}(t)$ is bounded uniformly in $t$. Moreover, (37) and the coercivity property show that $\partial_{t} \rho_{\varepsilon}$ is a finite measure whose total mass is uniformly bounded in each bounded interval $\left[t_{0}, t_{1}\right] \subset(0,+\infty)$. By the Arzela-Ascoli theorem we can extract a subsequence $\rho_{\varepsilon_{n}}$ such that $\rho_{\varepsilon_{n}}(t) \rightarrow \rho(t)$ for every $t \geq 0$. The estimates (37) and (45) show that for every $t>0, \rho(t)$ belongs to the domain of the quadratic forms $\mathfrak{a}$ and $\mathfrak{b}$, and satisfies a similar estimate

$$
\frac{1}{2} \mathfrak{b}(\rho(t))+t \mathfrak{a}(\rho(t))+t^{2} \mathfrak{b}\left(\partial_{t} \rho(t)\right) \leq \frac{1}{2} \liminf _{\varepsilon \downarrow 0} \mathfrak{b}\left(\rho_{\varepsilon}^{0}\right)<+\infty
$$

Let $\sigma \in \mathscr{M}(\bar{D})$ be an arbitrary element of the domains of $\mathfrak{a}$ and $\mathfrak{b}$; by 47 we can find a family $\sigma_{\varepsilon}$ (actually a family $\sigma_{\varepsilon_{n}}$, but we suppress the subscript $n$ ) weakly converging to $\sigma$ such that 46) holds. By (34) we have

$$
\mathfrak{b}_{\varepsilon}\left(\rho_{\varepsilon}(t), \sigma_{\varepsilon}\right)+\int_{0}^{t} \mathfrak{a}_{\varepsilon}\left(\rho_{\varepsilon}(r), \sigma_{\varepsilon}\right) \mathrm{d} r=\mathfrak{b}_{\varepsilon}\left(\rho_{\varepsilon}^{0}, \sigma_{\varepsilon}\right)
$$

and 37 with the Schwarz inequality yields the uniform bound

$$
\left|\mathfrak{a}_{\varepsilon}\left(\rho_{\varepsilon}(t), \sigma_{\varepsilon}\right)\right| \leq t^{-1 / 2} \mathfrak{b}_{\varepsilon}\left(\rho_{\varepsilon}^{0}\right)^{1 / 2} \mathfrak{a}_{\varepsilon}\left(\sigma_{\varepsilon}\right)^{1 / 2} \leq C t^{-1 / 2}
$$

where $C$ is independent of $\varepsilon$; we can therefore pass to the limit in $\sqrt{73}$ by Corollary 6 to find

$$
\mathfrak{b}(\rho(t), \sigma)+\int_{0}^{t} \mathfrak{a}_{\varepsilon}(\rho(r), \sigma) \mathrm{d} r=\mathfrak{b}\left(\rho_{0}^{0}, \sigma\right),
$$

so that $\rho$ is a solution of the limit equation. Since the limit is uniquely identified by the non-degeneracy and density condition 1 ), we conclude that the whole family $\rho_{\varepsilon}$ converges to $\rho$ as $\varepsilon \downarrow 0$. In particular $\rho$ satisfies the identity

$$
\frac{1}{2} \mathfrak{b}(\rho(t))+\int_{0}^{t} \mathfrak{a}(\rho(r)) \mathrm{d} r=\frac{1}{2} \mathfrak{b}\left(\rho^{0}\right) \quad \text { for every } t \geq 0
$$

This concludes the proof of 70 (and of Theorem 1).

In order to prove (71) (and Theorem 2 we note that by 35 and $\sqrt{74}$ we easily get

$$
\limsup _{\varepsilon \downarrow 0} \frac{1}{2} \mathfrak{b}_{\varepsilon}\left(\rho_{\varepsilon}(t)\right)+\int_{0}^{t} \mathfrak{a}_{\varepsilon}\left(\rho_{\varepsilon}(r)\right) \mathrm{d} r \leq \frac{1}{2} \mathfrak{b}(\rho(t))+\int_{0}^{t} \mathfrak{a}(\rho(r)) \mathrm{d} r
$$

The lower-semicontinuity property 45 and Fatou's Lemma yield

$$
\lim _{\varepsilon \downarrow 0} \mathfrak{b}_{\varepsilon}\left(\rho_{\varepsilon}(t)\right)=\mathfrak{b}(\rho(t)), \quad \lim _{\varepsilon \downarrow 0} \int_{0}^{t} \mathfrak{a}_{\varepsilon}\left(\rho_{\varepsilon}(r)\right) \mathrm{d} r=\int_{0}^{t} \mathfrak{a}(\rho(r)) \mathrm{d} r \quad \text { for every } t \geq 0 .
$$

Applying the same argument to (36) and its " $\varepsilon=0$ " analogue we conclude that $\mathfrak{a}_{\varepsilon}\left(\rho_{\varepsilon}(t)\right) \rightarrow$ $\mathfrak{a}(\rho(t))$ for every $t>0$.

Remark (More general ambient spaces). The particular structure of $\mathscr{M}(\bar{D})$ did not play any role in the previous argument, so that the validity of the above result can be easily extended to general topological vector spaces (e.g. dual of separable Banach spaces with their weak-* topology), once the coercivity condition of Lemma 10 is satisfied. 


\section{References}

[1] S. Adams, N. Dirr, M. A. Peletier, and J. Zimmer. Foundation of the Wasserstein gradient-flow formulation of diffusion: A large-deviation approach. In preparation.

[2] L. Ambrosio, N. Gigli, and G. Savaré. Gradient Flows in Metric Spaces and in the Space of Probability Measures. Lectures in mathematics ETH Zürich. Birkhäuser, 2005.

[3] R. Aris. Mathematical Modeling: A Chemical Engineer's Perspective. Academic Press, 1999.

[4] H. Attouch. Variational convergence for functions and operators. Pitman (Advanced Publishing Program), Boston, MA, 1984.

[5] H. Brezis. Opérateurs maximaux monotones et semi-groupes de contractions dans les espaces de Hilbert. North Holland, 1973.

[6] H. Brezis. Analyse fonctionelle - Théorie et applications. Collection Mathématiques appliquées pour la maîtrise. Masson, first edition, 1983.

[7] G. Da Prato and A. Lunardi. On a class of self-adjoint elliptic operators in $L^{2}$ spaces with respect to invariant measures. J. Differential Equations, 234(1):54-79, 2007.

[8] G. Dal Maso. An introduction to $\Gamma$-convergence, volume 8 of Progress in Nonlinear Differential Equations and Their Applications. Birkhäuser, Boston, first edition, 1993.

[9] P. Érdi and J. Tóth. Mathematical Models of Chemical Reactions: Theory and Applications of Deterministic and Stochastic Models. Manchester University Press, 1989.

[10] J. E. Hutchinson. Second fundamental form for varifolds and the existence of surfaces minimising curvature. Indiana Univ. Math. J., 35:45-71, 1986.

[11] R. Jordan, D. Kinderlehrer, and F. Otto. The variational formulation of the FokkerPlanck Equation. SIAM Journal on Mathematical Analysis, 29(1):1-17, 1998.

[12] H. A. Kramers. Brownian motion in a field of force and the diffusion model of chemical reactions. Physica, 7(4):284-304, 1940.

[13] J. Lions and E. Magenes. Non Homogeneous Boundary Value Problems and Applications, volume I. Springer, New York-Heidelberg, 1972.

[14] A. Mielke. Energy-drift-diffusion equations with recombination as entropy-gradient system. In preparation, 2009.

[15] F. Otto. The geometry of dissipative evolution equations: The porous medium equation. Comm. PDE, 26:101-174, 2001.

[16] M. Pennacchio, G. Savaré, and P. Colli Franzone. Multiscale modeling for the bioelectric activity of the heart. SIAM J. Math. Anal., 37(4):1333-1370 (electronic), 2005.

[17] J. G. Rešetnjak. The weak convergence of completely additive vector-valued set functions. Sibirsk. Mat. Z̆., 9:1386-1394, 1968. 